\title{
Community integration among individuals with stroke: a scoping review protocol
}

\author{
Sanjana Shirish Tipnis ${ }^{1} \cdot$ Vaishnavi Vilas Pawar $^{1} \cdot$ Rahul Arun Shinde $^{1} \cdot$ Deeksha Kumari $^{1} \cdot$ Lavanya Padmashali $^{1}$. \\ Shashank Mehrotra ${ }^{1}$ (i)
}

Received: 14 August 2020 / Accepted: 18 May 2021

(C) The Author(s) 2021

\begin{abstract}
Objective Successful community integration involves active participation in the community, broadly defined as having independence, relationships, and engagement in meaningful activities. This review aims to identify the literature focusing on community integration of individuals with stroke aged 18 years and older in the community over the past two decades.

Methods This review will be based on the methodological framework given by Arksey and O'Malley (2005). We will include studies from SCOPUS, PubMed, Web of Science, Google Scholar, CINAHL, EBSCO, ClinicalKey, Wiley Online, ProQuest, and Cochrane Library databases published from January 2000 onwards. Both quantitative and qualitative data will be analyzed separately to identify various assessments, interventions, and factors that influence the community integration of individuals with stroke.

Conclusion This scoping review will help to explore studies focusing on community integration among individuals with stroke aged 18 and older living in the community. It can further assist professionals who are addressing the concerns of individuals with stroke in the community.
\end{abstract}

Keywords Community integration $\cdot$ Occupational therapy $\cdot$ Stroke $\cdot$ Community participation

\section{Introduction}

Stroke is among the leading causes of disability among adults (Mozaffarian et al. 2015). It reportedly accounts for 17.9 million deaths each year worldwide, with a particularly high burden in low- and lower-middle-income countries (WHO 2020). Among adults aged 35 to 64 years, mortality due to stroke increased from 14.7/100,000 in 2010 to $15.4 / 100,000$ in 2016 (Virani et al. 2021). The wide array of impairments and complexity of stroke results in severe disability, which may be associated with early retirement (Alavinia and Burdorf 2008). Such impairments disrupt daily activities, relationships, and expectations of the future (Wallenbert and Jonsson 2005). These losses influence individuals' ability to participate in everyday occupations across their life span (Arntzen et al. 2015).

Shashank Mehrotra

shashank.mehrotra@manipal.edu

1 Department of Occupational Therapy, Manipal College of Health Professions, Manipal Academy of Higher Education, Manipal, Udupi, Karnataka 576104, India
Participation or integration in a community is an essential goal of rehabilitation. In stroke rehabilitation, the transition from acute care to inpatient rehabilitation to community rehabilitation is a sign of progressive recovery (Lindsay et al. 2014).

Poor community integration affects the functioning of stroke survivors. With an emphasis on this, it is vital to understand how community integration can be a targeted outcome in interventions for individuals with stroke (WHO 2001). Successful community integration involves active participation in the community, broadly defined as having independence, relationships, and engaging in meaningful activities. It is seen as an opportunity to live in the community and be valued for one's uniqueness and abilities, like everyone else (Salzer and Baron 2006). In community-based clinical practice, it is important to learn about various factors that influence community integration and interventions used for stroke recovery in community rehabilitation services. For this review, we will consider community integration as an intervention that is focused on improving an individual's participation in three main areas: paid employment or other productive activity, independent living, and social activities.

Community-based rehabilitation is a critical component of recovery to help patients regain lost skills and relearn tasks 
and thus regain independence. The principles of intervention to enable community integration are directed toward the barriers to employment after stroke (Treger et al. 2007) such as mood, working memory (Hommel et al. 2009), fear of deterioration (McLean 2007; Alaszewski et al. 2007), architectural barriers, weak local economy, transportation challenges, and stereotyping of individual with disabilities. Post-stroke rehabilitation helps patients to achieve community integration and self-sufficiency in employment. Therefore, developing a framework of rehabilitation goals and interventions for efficient outcomes is essential (Dobkin and Dorsch 2013).

Rehabilitation for individuals with stroke focuses mainly on community-based interventions that aim to enhance an individual's participation in daily living (Rijken and Dekker 1998). The outcome of an intervention may focus on improving the engagement of patients in the community (Arntzen et al. 2015). The rehabilitation should continue until community integration is achieved. Initially, rehabilitation for stroke should concentrate on education and prevention of complications, and later focus on the long-term rehabilitation plan. After dealing with this crucial aspect of self-care, mobility, communication, and homemaking, the further focus of the intervention should be on social competency and participation, community mobility, and financial and health management. Further rehabilitation of an individual with stroke should progress to employment and education (Duncan et al. 2005).

Previous studies (Walsh et al. 2015, Hui et al. 2021) have revealed a gap in rehabilitation research on community integration of individuals with stoke, and have emphasized the need to focus on the re-integration of individuals with stroke in paid occupations as part of their rehabilitation program. Thus, this scoping review is planned to explore and summarize the available rehabilitation literature on the subject. It will provide professionals an overview of various available assessments and rehabilitation interventions for individuals with stroke in the community and the factors influencing them. This information will in turn help to bridge the current gap between patients' independence and participation, resulting in greater inclusion of individuals with stroke in the community.

Aim This scoping review aims to identify the available literature focusing on community integration (employment/other productive activities, independent living, social participation) of individuals with stroke aged 18 years and older in the last two decades (January 2000 onwards).

\section{Objective}

1. To provide an overview of various studies related to community integration (employment/other productive activities, independent living, social participation) among individuals with stroke aged 18 years and older.

2. To provide a summary of various assessments and interventions used by professionals to facilitate community integration among individuals with stroke.

3. To determine what factors influence the interventions provided to facilitate community integration in individuals with stroke.

\section{Method and analysis}

We will follow the method informed by Arksey and O'Malley's (2005) methodological framework and other studies (Georlee et al. 2020). This scoping review aims to identify the literature from January 2000 onwards focusing on community integration (employment/other productive activities, independent living, social participation) of individuals with stroke in the community. Table 1 depicts the stages of the scoping review process, which are described in more detail in the subsequent sections.

\section{Developing a research question}

A broad research question was developed to ensure that the scoping review would not only include a particular type of studies, but would also be able to capture the diversity and scope of literature available. The previous section depicts the developed objectives for this scoping review.

Research question What is the existing literature focusing on community integration of individuals with stroke aged 18 years and older in the community?

\section{Study identification}

The search will identify data from SCOPUS, PubMed, Web of Science, Google Scholar, CINAHL, EBSCO, ClinicalKey, Wiley Online, ProQuest, and Cochrane Library electronic databases. We will search the reference lists of the articles to ensure the inclusion of all relevant literature. Studies will be selected according to the criteria listed in Table 2. We selected the databases in consultation with a subject expert to ensure proper identification of all relevant literature articles. We will conduct an initial search of a selection of related databases, followed by the title and abstract screening by

Table 1 Methodological framework
Step 1: Identifying the research question

Step 2: Identifying relevant studies

Step 3: Study selection

Step 4: Charting the data

Step 5: Collating, summarizing, and reporting the scoping review results 
Table 2 Study selection criteria

\begin{tabular}{ll}
\hline Inclusion criteria & Exclusion criteria \\
\hline $\begin{array}{c}\text { All available articles with community integration focusing on three main areas: } \\
\text { employment/other productive activities, independent living, and social } \\
\text { participation }\end{array}$ & $\begin{array}{c}\text { Conference proceedings } \\
\text { and abstracts }\end{array}$ \\
All available studies including a group of people diagnosed with stroke (either \\
acute, subacute, or chronic, having any physical or cognitive disability) aged \\
$\begin{array}{l}18 \text { and older living in the community } \\
\text { All peer-reviewed, non-peer-reviewed, and grey literature articles in the English } \\
\text { language published from January 2000 onwards }\end{array}$ \\
\hline
\end{tabular}

two independent reviewers. Also, we will analyze the text articles and keywords used in the articles. Articles that do not meet the inclusion criteria will be removed. Through a systematic search of Medical Subject Headings (MeSH) terms on MEDLINE, we selected the keywords that will be utilized to search the articles.

We conducted an initial preliminary screening to avoid using broad searches that would result in an unmanageably large number of results. Also, the results of the initial preliminary screening were discussed with a subject expert to refine and finalize the search string (Table 3). The cutoff date to be applied for the search strategy screening will be 28 February 2021. Besides, the reference lists of the included articles will be searched to ensure that all relevant studies are included.

\section{Study selection for the review}

We will strictly limit the search terms used. There will be no restrictions by country for the initial screening. Studies published within the past two decades (January 2000 onwards) will be included in the review. We will use additional database-specific filters including time span and age groups to obtain clear information and to avoid unmanageably vast search results obtained in the full search.

We have chosen the preliminary inclusion and exclusion criteria as a general guideline to select the studies for the review. Therefore, we will include all the studies that meet our inclusion criteria regardless of the quality and rigor of the research. We consulted a subject expert to determine the final databases and search strategies. All imported citations will be uploaded into the Mendeley platform to manage the project. Mendeley will be used to upload the abstracts and

Table 3 Keywords derived from the Medical Subject Headings terms (MeSH)

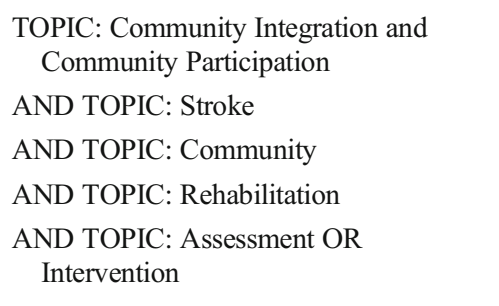

titles captured in the initial screening. Two independent reviewers $(\mathrm{ST})$ and $(\mathrm{V})$ will screen all imported literature to determine its eligibility for selection into the scoping review. In case of any disagreement, another reviewer will be involved until we get a consensus. Full-texts of the articles will be uploaded for the articles that meet the inclusion criteria. For this scoping review, we will use the Preferred Reporting Items for Systematic Reviews and Meta-Analyses Protocols (PRISMA-P) flow diagram to document the search and visually represent all the studies identified in each step of the study selection process.

\section{Charting the data for scoping review}

We will use a descriptive-analytical method to ensure that collected information can be disseminated in a concise format. This approach will assist the researchers in identifying previous data that will answer the research question, organizing the current literature, and identifying significant themes and patterns within the literature. We will ensure proper care when charting the data to maintain accuracy.

Quantitative and qualitative data will be analyzed to identify the factors that influence the community integration of individuals with stroke. The primary researcher (ST) and secondary reviewer (SM) will independently identify themes and establish subthemes from the data sources included in the review. Following this, they will together discuss the themes and subthemes without debating in the case of disagreement. A third reviewer (LP) will be separately approached to clarify any disagreements between the first two reviewers (ST and SM). Before grouping, themes and qualitative data will be described. Such practice will assist in identifying gaps in the existing literature.

We will extract and tabulate the following general information: author name(s), name of the journal, publication year, country, study methodology (population, aims/objectives/ question, research setting, and study-design), and findings. Data will also be extracted from the following areas: assessments, interventions, and factors influencing the community integration (employment/other productive activities, independent living, social participation) of individuals with stroke. 


\section{Summarizing, collating, and reporting the results of the scoping review}

We will present a descriptive and narrative summary of the study findings using summary tables to describe the components of each study. From the included research studies, key findings will be identified to describe community integration among individuals with stroke. The results of this scoping review will then be presented in an article having applications to community-based rehabilitation. This will also help professionals in exploring the knowledge of community integration among individuals with stroke and the factors influencing community integration.

Study timeline The proposed timeline of the study is described in Table 4.

\section{Patient and public involvement}

There will be no participation of any patients or the general public in the scoping review.

\section{Discussion}

To our knowledge, this scoping review examining the concept of community integration among individuals with stroke is the first of its kind. This review has the potential to improve the understanding among professionals caring for individuals with stroke. In this review, we aim to review and appraise the articles focusing on community integration in the areas of employment/other productive activities, independent living, and social participation among individuals with stroke aged 18 and older, various assessments and interventions

Table 4 Study timeline

Stage 1: Identifying the research question: The research questions have been formulated to develop the scoping review protocol and to initiate the planning of the scoping review to follow.

Stage 2: Identifying relevant studies: The databases and search strategy have been determined in consultation with a subject librarian. Relevant articles for this scoping review will be identified accordingly.

Stage 3: Study selection: Inclusion and exclusion criteria have been formulated. Based on these, we will decide on the inclusion of articles for the scoping review.

Stage 4: Charting the data: The data will be charted once step 3 has been completed in its entirety. The researchers will have 30 working days to conduct the descriptive analysis and to tabulate all the research findings.

Stage 5: Summarizing, collating and reporting the results of the scoping review: Once phase 4 has been completed, phase 5 will be initiated. The scoping review findings will be compiled in an article to ensure optimal dissemination of the results. available, and factors influencing the application of such interventions. This will give us a better understanding of patients' independence and participation resulting in community inclusion of individuals with stroke, which will inform further research, practice, and training.

\section{Ethics and dissemination}

As a secondary analysis, this scoping review does not require ethics approval. Results will summarize existing research related to community integration among individuals with stroke. We will further disseminate the results through peer-reviewed journals and conferences targeting clinicians, academics, and researchers.

Acknowledgements The authors would like to thank Dr. Sumita Rege (Associate Professor, Department of Occupational Therapy, Manipal College of Health Professions, Manipal Academy of Higher Education, for her support in reviewing the preliminary draft.

Authors' contributions Shashank Mehrotra and Sanjana Shirish Tipnis initiated the idea of this research, followed by discussion among all authors, which contributed to finalizing the research idea. Shashank Mehrotra and Sanjana Shirish Tipnis contributed to the conceptualization of the research title, aim, and objectives. Following this, four authors (Sanjana Shirish Tipnis, Vaishnavi Vilas Pawar, Rahul Arun Shinde, and Lavanya Padmashali) searched different electronic databases to understand the background of the research. This was supervised by the corresponding author, Shashank Mehrotra. The methodology was drafted by Deeksha Kumari and Shashank Mehrotra. It was further revised by Sanjana Shirish Tipnis and Lavanya Padmashali. Shashank Mehrotra and Lavanya Padmashali drafted the manuscript and revised it further.

Funding Open access funding provided by Manipal Academy of Higher Education, Manipal. This research received no funding from any agency in the public, commercial, or not-for-profit-sector.

Availability of data and materials Not applicable.

\section{Declarations}

Ethical approval This review study does not require ethics clearance, as we are taking only secondary data from the available literature.

Consent to participate There will be no participation of any patients or the general public in the scoping review.

Consent for publication Not applicable.

Competing interests The authors declare that have no conflict of interest.

Open Access This article is licensed under a Creative Commons Attribution 4.0 International License, which permits use, sharing, adaptation, distribution and reproduction in any medium or format, as long as you give appropriate credit to the original author(s) and the source, provide a link to the Creative Commons licence, and indicate if changes were made. The images or other third party material in this article are included 
in the article's Creative Commons licence, unless indicated otherwise in a credit line to the material. If material is not included in the article's Creative Commons licence and your intended use is not permitted by statutory regulation or exceeds the permitted use, you will need to obtain permission directly from the copyright holder. To view a copy of this licence, visit http://creativecommons.org/licenses/by/4.0/.

\section{References}

Arksey H, O’Malley L (2005) Scoping studies: towards a methodological framework. Int J Soc Res Methodol 8(1):19-32. https://doi.org/10. 1080/1364557032000119616

Alaszewski A, Alaszewski H, Potter J, Penhale B (2007) Working after a stroke: survivors' experiences and perceptions of barriers to and facilitators of the return to paid employment. Disabil Rehabil 29(24):1858-1869. https://doi.org/10.1080/09638280601143356

Alavinia SM, Burdorf A (2008) Unemployment and retirement and illhealth: a cross-sectional analysis across European countries. Int Arch Occup Environ Health 82(1):39-45. https://doi.org/10.1007/ s00420-008-0304-6

Arntzen C, Borg T, Hamran T (2015) Long-term recovery trajectory after stroke: an ongoing negotiation between body, participation and self. Disabil Rehabil 37(18):1626-1634. https://doi.org/10.3109/ 09638288.2014.972590

Duncan PW, Zorowitz R, Bates B, Choi JY, Glasberg JJ, Graham GD, Katz RC, Lamberty K, Reker D (2005) Management of adult stroke rehabilitation care: a clinical practice guideline. Stroke 36(9):e100 e143. https://doi.org/10.1161/01.STR.0000180861.54180.FF

Dobkin BH, Dorsch A (2013) New evidence for therapies in stroke rehabilitation. Curr Atheroscler Rep 15(6):331. https://doi.org/10.1007/ s11883-013-0331-y

Georlee GM, U A, Dat PN, Tuan NK, Mehrotra S (2020) Home modification interventions addressing falls and participation in activities of daily living among older adults: a scoping review protocol. BMJ Open 10:e039742. https://doi.org/10.1136/bmjopen-2020-039742

Hui YY, Vytialingam N, Singh SK (2021) Barriers to community integration for older people in Malaysia: a qualitative study from occupational therapist perspectives. Public Health Open J 6(1):1-8. https://doi.org/10.17140/PHOJ-6-152

Hommel M, Miguel ST, Naegele B, Gonnet N, Jaillard A (2009) Cognitive determinants of social functioning after a first ever mild to moderate stroke at vocational age. J Neurol Neurosurg Psychiatry 80:876-880. https://doi.org/10.1136/jnnp.2008.169672
Lindsay P, Furie KL, Davis SM, Donnan GA, Norrving B (2014) World stroke organization global stroke services guidelines and action plan. Int J Stroke 9:4-13. https://doi.org/10.1111/ijs.12371

McLean R (2007) Employment status six months after discharge from inpatient rehabilitation for a mild-to-moderate physical disability. Ann Acad Med Singap 36(1):18-21

Mozaffarian D et al (2015) Heart disease and stroke statistics - 2015 update: a report from the American Heart Association. Circulation 131:e29-e322. https://doi.org/10.1161/CIR.0000000000000152

Rijken PM, Dekker J (1998) Clinical experience of rehabilitation therapists with chronic diseases: a quantitative approach. Clin Rehabil 12(2):143-150. https://doi.org/10.1191/026921598669374346

Salzer MS, Baron RC (2006) Community integration and measuring participation. University of Pennsylvania Collaborative on Community Integration. Available online at, Philadelphia www. upennrrtc.org

Treger I, Shames S, Giaquinto S, Ring H (2007) Return to work in stroke patients. Disabil Rehabil 29(17):1397-1403. https://doi.org/10. 1080/09638280701314923

Virani SS, Alonso A, Aparicio HJ, Benjamin EJ, Bittencourt MS, Callaway CW, Carson AP, Chamberlain AM, Cheng S, Delling FN, Elkind MS (2021) Heart disease and stroke statistics-2021 update: a report from the American Heart Association. Circulation 143:e254-e743. https://doi.org/10.1161/CIR.0000000000000950

World Health Organization (WHO) (2001) International classification of functioning, disability, and health (ICF), WHO, Geneva. Retrieved from International Classification of Functioning, Disability and Health (ICF) (who.int) accessed on 10 June 2020

Wallenbert I, Jonsson H (2005) Waiting to get better: a dilemma regarding habits in daily occupations after stroke. Am J Occup Ther 59(2): 218-224. https://doi.org/10.5014/ajot.59.2.218

Walsh ME, Galvin R, Loughnane C, Macey C, Horgan NF (2015) Community re-integration and long-term need in the first five years after stroke: results from a national survey. Disabil Rehabil 37(20): 1834-1838. https://doi.org/10.3109/09638288.2014.981302

World Health Organization (WHO) (2020), World health statistics 2020: monitoring health for the SDGs, sustainable development goals. Geneva. Retrieved from World health statistics 2020: monitoring health for the SDGs, sustainable development goals (who.int) accessed on 10 July 2020

Publisher's note Springer Nature remains neutral with regard to jurisdictional claims in published maps and institutional affiliations. 